\title{
Variability in echolocation call design of 26 Swiss bat species: consequences, limits and options for automated field identification with a synergetic pattern recognition approach
}

\author{
Martin K. OBRIST \\ Ruedi BOESCH
}

Swiss Federal Research Institute WSL, Research Department Landscape

$\mathrm{CH}-8903$ Birmensdorf (Switzerland)

martin.obrist@wsl.ch

ruedi.boesch@wsl.ch

Peter F. FLÜCKIGER

Bat Protection Kt SO. c/o Museum of Natural History Olten, Kirchgasse 10, $\mathrm{CH}-4600$ Olten (Switzerland) info@naturmuseum-olten.ch

KEY WORDS

Echolocation, pattern recognition, Chiroptera, monitoring, Switzerland.
Obrist M. K., Boesch R. \& Flückiger P. F. 2004. - Variability in echolocation call design of 26 Swiss bat species: consequences, limits and options for automated field identification with a synergetic pattern recognition approach. Mammalia 68 (4): 307-322.

\begin{abstract}
Pattern recognition algorithms offer a promising approach to recognizing bat species by their echolocation calls. Automated systems like synergetic classifiers may contribute significantly to operator-independent species identification in the field. However, it necessitates the assembling of an appropriate database of reference calls, a task far from trivial. We present data on species specific flexibility in call parameters of all Swiss bat species (except Nyctalus lasiopterus and Plecotus alpinus). The selection of "training-calls" for the classifier is crucial for species identification success. We discuss this in the context of echolocation call variability differing between species and its consequences for the implementation of an automated, species specific bat activity monitoring system.
\end{abstract}


MOTS-CLÉS

Echolocation, signaux,

Chiroptera, reconnaissance automatique,

\section{RÉSUMÉ}

Variabilité des signaux d'écholocation de 26 espèces de chauves-souris suisses: conséquences, limites et options pour un système automatisé d'identification avec une approche de reconnaissance synergique.

Les algorithmes de reconnaissance de patron représentent une approche prometteuse pour identifier les espèces de chauves-souris à partir de leurs signaux d'écholocation. Des systèmes automatisés tels que les classifications synergiques peuvent contribuer à une identification sur le terrain indépendamment de l'expérience de l'opérateur. Cependant il est nécessaire de constituer une solide base de données de signaux de référence, une tâche délicate. Cet article présente des données sur la variabilité des paramètres des signaux de toutes les espèces de chauves-souris suisses (à l'exception de Nyctalus lasiopterus et Plecotus alpinus). La sélection des "signaux d'apprentissage " est déterminante pour une bonne identification des espèces. Ceci est discuté dans le contexte d'une variabilité des signaux d'écholocation, différente selon les espèces. Les conséquences de ces résultats sont évaluées dans la perspective de réalisation d'un système automatisé de suivi d'activité spécifique des chauves-souris.

\section{INTRODUCTION}

Due to their active orientation system, echolocating bats are conspicuous. Therefore, by acoustically monitoring bats we can eavesdrop on their behaviour. In the course of evolution, bats have occupied the nocturnal niche of almost every possible habitat. In parallel with their evolution, wing morphology, hearing abilities and echolocation call characteristics adapted to the specific habitats and foraging method (Neuweiler 1999). Today signal variability covers constant frequency and frequency modulated calls of various composition and temporal structure. Additionally, bats adapt their calls to the particular situation, e.g., using short, broad-band signals at high repetition rate when hunting close to clutter, or long narrow-band signals when foraging in open air space (Kalko 1995).

Despite this flexibility, recognition of bat species by their calls can be tackled. This is an old theme with new variations. In 1958 already, Griffin described echolocation calls differing between species (Griffin 1958). But it was not until 23 years later, when the first publications dedicated to acoustic bat species identification where published by Ahlén (1981), and Fenton \& Bell (1981).

The topic gained new momentum in recent years in the light of affordable biodiversity monitoring and conservation. Advances in technology further facilitate registering and analysing of ultrasound signals (Parsons and Obrist 2004). Different approaches can be taken to quantitatively or qualitatively analyse echolocation signals (Parsons et al. 2000).

Heterodyning detectors look at a narrow frequency band. Calls with differing energy content or different temporal sweep structure in this frequency band will sound differently in such a detector (Fig. 1). Set to $45 \mathrm{kHz}$, a Pipistrellus pipistrellus will be easily detected as strong plop, while Vespertilio murinus will probably not be heard at all and Eptesicus serotinus might just be audible as weak tick. Species with short, broadband signals will mostly tick very similar, possibly with different intensity depending on the energy 


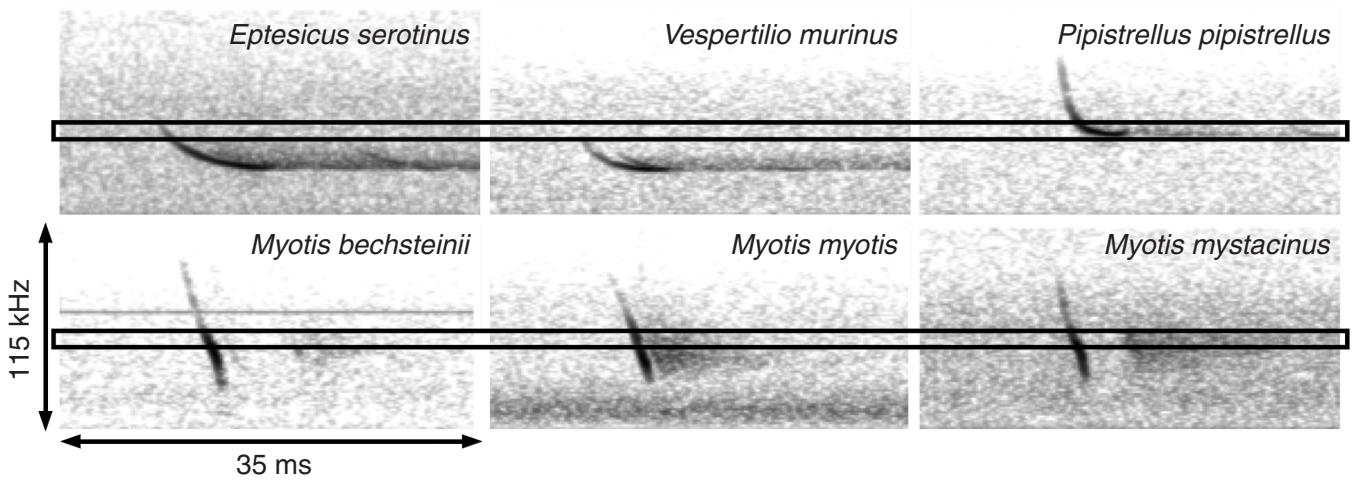

FIG. 1. - Symbolic representation of the acoustic filter which a heterodyning bat detector implies. Echolocation calls of six species are given as spectrograms. The central black rectangle enframes the few $\mathrm{kHz}$ around $45 \mathrm{kHz}$, at which the detector is tuned. Eptesicus serotinus will be heard as weak "tick", Vespertilio murinus will not be heard at all and Pipistrellus pipistrellus will sound as full "tock". The three Myotis species will all sound very similarly as loud short "tick".
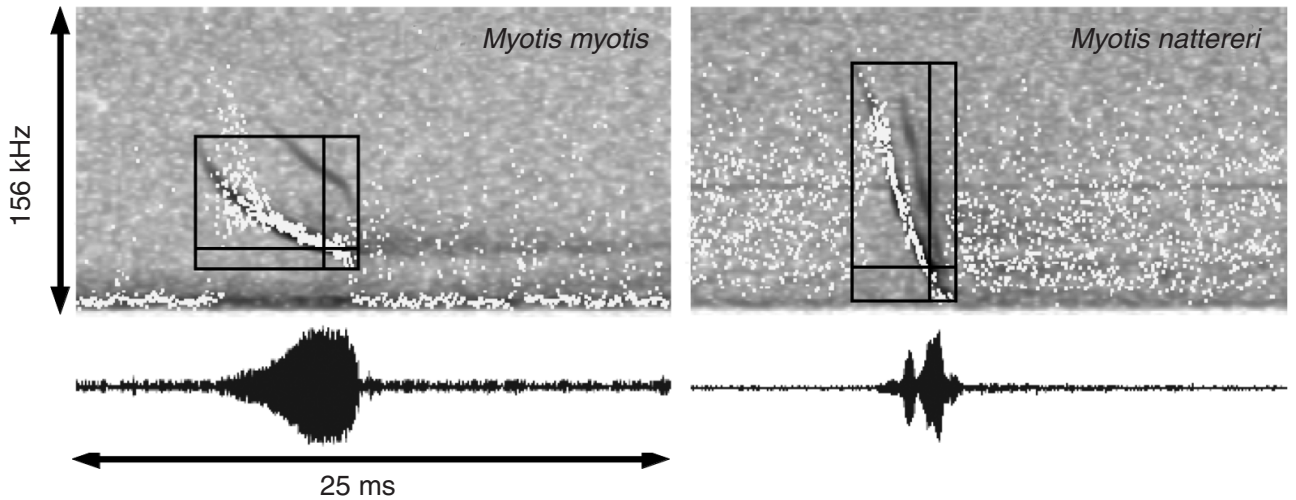

FIG. 2. - Spectrographic (dark grey) and zero-crossing (white dots) representation of echolocation calls of Myotis myotis and Myotis nattereri. Period-plots (zero-crossing displays) do not show harmonics and dots may "jump" between harmonics of similar intensity (see middle part of call of Myotis nattereri). The display becomes random for low intensity signals (beginning of calls). - The black frame indicates parameters measured in Canary for statistical analysis: duration, highest and lowest frequency. The intersection of the black cross indicates the measurement of the frequency of peak energy.

maximum of their calls and their repetiton rates. Interpretation and valuation of all these parameters heavily depend on the experience of the observing person.

A visual approach to echolocation call identification can be taken by either calculating spectrograms or period-plots (Fig. 2). The latter is achievable in real time with a limited amount of hardware (e.g., ANABAT system, Titley
Electronics 1998; O’Farrell et al. 1999a), while spectrogram calculation usually necessitates a fast computer. However, period- or zero-crossing plots do perform badly with weak or noisy signals, blurring the display considerably (Fig. 2). This complicates the recognition in some situations and species, and their use is discussed very controversially (Barclay 1999; O'Farrell et al. 1999b; Fenton 2000; Fenton et al. 2001). 
If real-time performance is not required, offline analysis offers more accurate methods to species recognition. It involves in most cases interaction with an operator, who measures in the amplitude or the spectrogram display critical parameters like highest and lowest frequency, frequency of main energy and duration of the signal (Fig. 2, black frames with cross). With these discrete measurements of spectral and temporal parameters, further analyses with statistics, artificial neural networks or decision trees can be performed, giving good performance (Herr et al. 1997; Parsons \& Jones 2000; Russo \& Jones 2002; Obrist et al. 2004).

Still, in many cases, measurements will be identical for calls from different species. In some of these cases, the spectrogram may show characteristic signatures in the shape of the frequency sweep. Here, a pattern recognition approach using shape analysis may help to discriminate signals.

In a pilot study, Obrist et al. (2004) had analyzed echolocation signals of 12 species. With a less advanced recording setup and a limited data set, they had reached average recognition rates of $80 \%$. Here we report on data of 26 species, which were all recorded directly to digital media. We tested the hypothesis, that a pattern recognition approach based on a synergetic algorithm will outperform classical statistical analysis of parametric measurements, even for larger species assemblages.

\section{MATERIAL AND METHODS}

\section{RECORDING}

All recordings were done with a Pettersson D980 bat detector connected through a voltage amplifier stage to a PCMCIA data acquisition card (ComputerBoards PC-CARD-DAS16/330) in an Apple Macintosh PowerBook laptop computer (PowerBook 3400 or PowerBook G3) and driven by custom made software. Thus, digital recordings of 26 bat species' echolocation calls where acquired, mostly when releasing identified bats, rarely in front of previously inspected roosts of known species occupancy. Tadarida teniotis was recorded in free hunting flight.

643 sequences (3.6 hours), containing calls of 362 hand-identified specimens were recorded. Prior to analysis, high-pass filtering $(7.5 \mathrm{kHz})$ was applied and single echolocation calls $(26 \mathrm{~ms}$ $=8192$ data points) were cut from the sequences. Most species' calls fit into this window size (except Rhinolophidae) and only rarely there were more than one signal enclosed in a single cutout.

\section{SigNAL ANALYSIS AND STATISTICS}

52302 signals were cut from the recordings by help of a simple integrating detector algorithm. 37948 of these were visually qualified as noise or otherwise inadequate for the analysis. Of the remaining 14354, 5153 where qualified suitable for identification purposes and 9201 additionally as suitable for training. Of these in a randomly selected subset of 2398 calls we calculated high resolution spectrograms $(0.6 \mathrm{kHz}$ resolution, $87.5 \%$ window overlap) with Canary (Cornell University, Ithaca). Duration (DUR), highest frequency (HFR), lowest frequency (LFR) and frequency of main energy (MFR) were then manually extracted for later statistical analysis with a discriminant component analysis with resubstitution (PROC DISCRIM) in SAS (SAS Institute Inc.). The discriminant function was ten times repeated with $12.5 \%, 25 \%$ or $50 \%$ of all 2398 considered calls and tested with the remaining ones. Percentage values of coefficients of variation $(\mathrm{CV})$ were transformed $\arcsin (\sqrt{C V})$ for statistical comparison (Zar 1984).

\section{SyNERGETICS}

We apply a synergetic algorithm, which is also used e.g., in product control (Haken, 1988; Wagner et al. 1993; Wagner et al. 1995; Haken 1996). The classification of bat calls is achieved with an algorithm termed SC-MELT (Wagner et al. 1995; Hogg \& Talhami 1996; Dieckmann 1997). This algorithm combines several training patterns per class into one feature vector, which has the same dimension as the training vectors. This ability enables the synergetic algo- 
rithm to handle big dimensions in contrast to artificial neural networks (ANN). We calculated spectrograms each consisting of 159128 point-spectra for the pattern recognition approach. The computational power needed to train an ANN with an input vector of size 20352 (spectrogram of dimension $159 \times 128$ ) is prohibitive.

The synergetic algorithm emphasizes unique pattern content of training signals and diminishes of pattern contents common to all others. Learning times are very fast: the generation of the prototypes containing hundreds of bat calls takes only a couple of minutes on a current G4 processor (Apple Macintosh Power Mac G4/867). The classification of a signal is even faster because it is simply a scalar product. Each signals classification result consists of an array of scalar product values, which reflects the signals match to each stored class (i.e. the 26 acquired bat species).

First, we performed learning tests on random subsets of 520 echolocation calls, 20 calls per each of the 26 species. The random sets were issued from the full database of 9201 signals which where visually approved as good for learning purposes, i.e. which did not contain e.g., calls immediately after take-off or strong echoes. We tested against a second set of 520 different signals from the same database.

We performed three selections of learning calls and tested each training set against three other selections of classification calls.

Apart from the raw classification rates we also calculated recognition rates with a filter criteria, which rejected classifications with maximum scalar product values smaller than 0.6 or with a difference to the next best scalar product of less than 0.2 .

We then tried to optimize the training base by picking from every species the calls from those random sets, which had achieved the highest classification rates. Thus, we generated a fourth training base consisting of a simplistically optimized sub-selection. Again we tested the three classification sets against this training base.

\section{RESULTS}

\section{STATISTICS}

\section{Echolocation call parameters}

Figures 3 and 4 illustrate the range of echolocation calls considered in this study for every investigated species. The results of the parametric spectrogram measurements are given in Table 1. For further analysis we split the species in two groups: the genus Myotis and all other species. Myotis have significantly shorter signals (DUR) with significantly higher starting frequencies (HFR; Table 1, t-test, the columns below the mean values).

To test the within-species variability against the between-species variability of echolocation call parameters, we calculated the coefficients of variation (CV) for every species and parameter. We then compared CVs averaged over all Myotisspecies against CVs averaged over all other species with a t-test. Both groups show comparable within-species variation in the duration and highest frequency of their calls. However, Myotis species show significantly higher variation in the lowest frequency and the frequency of peak energy (Table 1, t-test, the columns below the $\mathrm{CV}$ values).

To compare between-species variation we performed a variance ratio test, again between Myotis and non-Myotis species. Here, the latter show significantly higher variance in all four parameters compared to the genus Myotis (Table 1, Variance ratio test). Thus, variation within Myotis species is larger than in other species, but species differ less among each other relative to the non-Myotis species.

\section{Discriminant function analysis}

With discriminant function analyses (DFA) we explored the classification power of parametric measurements to be able to compare against the power of the pattern recognition approach. Increasing the number of calls to calculate the function did result in a general increase of the percentage of correctly reclassified calls from $68 \%$ to $75 \%$. However, for Myotis bechsteinii and Myotis brandtii a decreased classification success 


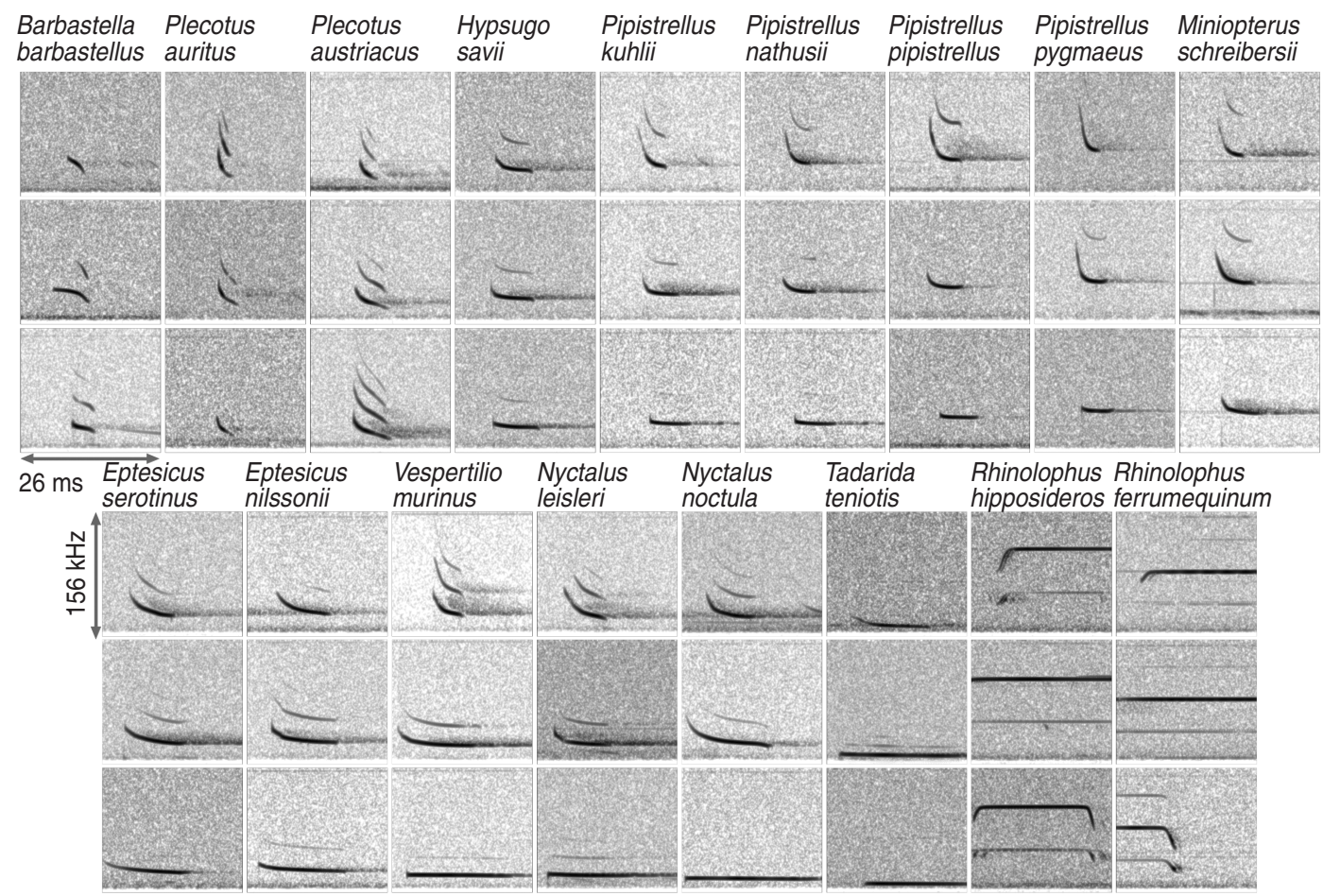

FIG. 3. - Spectrograms of three exemplary echolocation calls of each investigated bat species not belonging to the genus Myotis. In many species signals show high plasticity, ranging from broad-band, multiharmonic calls to very narrow-band, quasi-constantfrequency vocalizations (e.g., Pipistrellus sp., Vespertilio murinus). Most signals in the Rhinolophidae where truncated by the chosen window length (see methods).

was observed $(33 \%$ to $31 \%$ and $33 \%$ to $28 \%$ respectively).

The averaged results of 10 DFA, calculated with equal numbers of data calls and test calls are given in Table 2. Reclassification results varied from $27 \%$ to $100 \%$. Species of the genus Myotis always scored considerably worse than did the other species.

\section{SYNERGETICS}

Table 3 summarizes the classification results for different settings. The fusion of a sub-selection of well-performing training calls to a new training base increased the average classification rate from $70 \%$ to $73 \%$ for raw classifications and from $86 \%$ to $88 \%$ in filtered classifications. Filtering lead to a rejection of $37 \%$ of the calls of the random set. The sub-selection slightly decreased this number to $31 \%$. Even though a reduced number of signals qualify for classification, the filtering approach improves classification rates by 12 $13 \%$.

Figure 5 illustrates the percentage of correct classifications with and without filter. The effect differs strongly between species but is never negative. Myotis species consistently show lower classification rates than other species.

\section{DISCUSSION}

\section{ECHOLOCATION CALLS}

Digital recording at $312.5 \mathrm{kHz}$ with 12 bit data depth ensured a high fidelity of the recordings (Parsons and Obrist 2004). The description and illustration of echolocation call characteristics of 


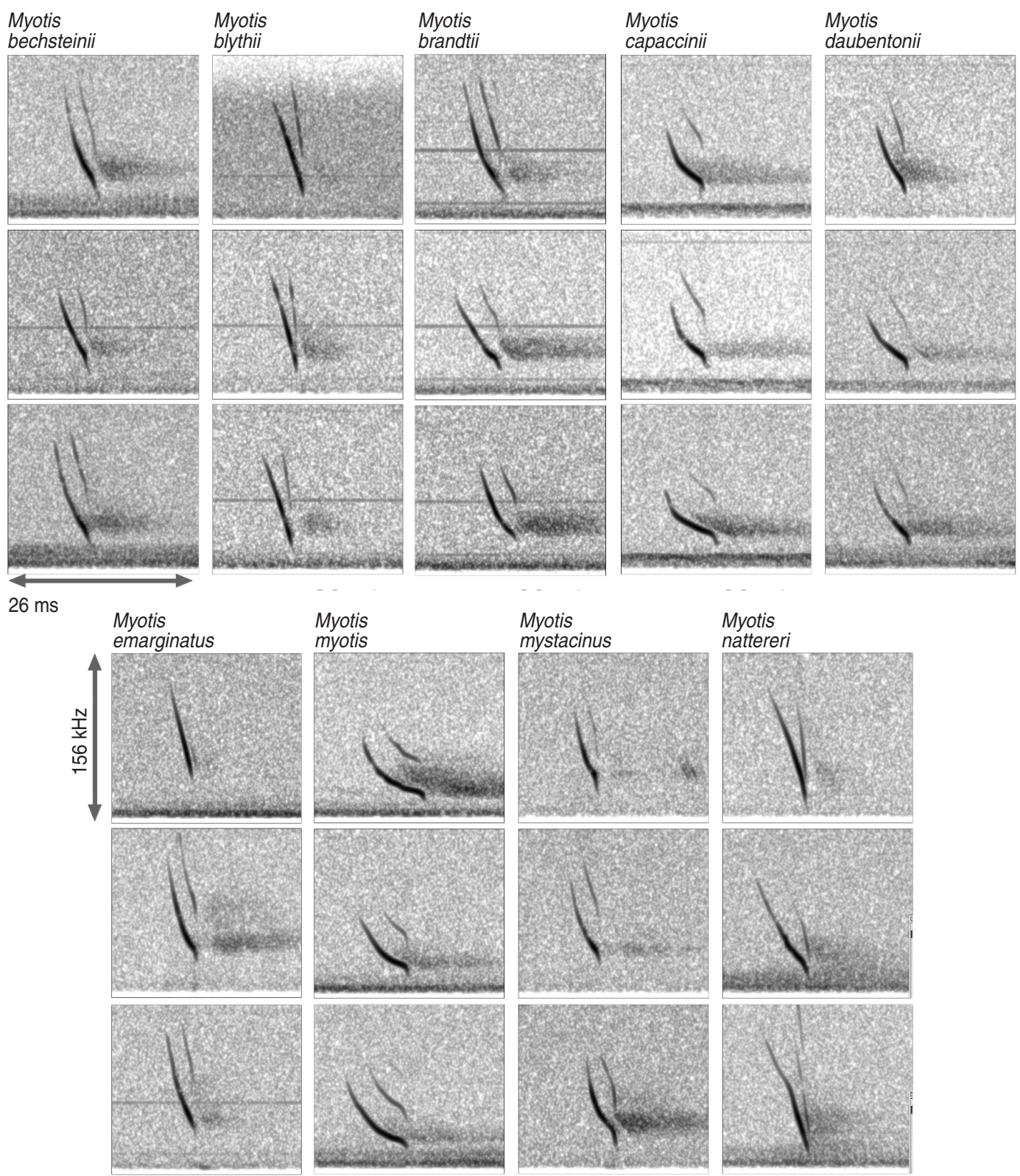

FIG. 4. - Spectrograms of three exemplary echolocation calls of each investigated bat species in the genus Myotis. Variability between species is considerably lower compared to Fig. 4.

26 Swiss bats species given here compare well with other published reports (e.g., Ahlén, 1981). Recordings made directly after releasing handidentified bats differ from those recorded later in search flight. By recording sequences of 20 seconds in a post-trigger mode and by monitoring the acoustic behaviour of the animals, we could verify and later select the type of calls contained 
TABLE 1. - Echolocation call parameters of 26 swiss bat species. Mean, standard deviation (StdDev) and coefficient of variation (CV) are given for call duration (DUR), lowest call frequency (LFR), frequency of peak energy (PFR) and highest frequency (HFR). Values given in italics do not properly indicate species' parameter, as the measurement window of 26 ms was truncating total call lengths. Range, mean and variances averaged over all species of the genus Myotis and over all other species are indicated below. Results of t-tests and variance ratio tests are given at the bottom (see text for further details).

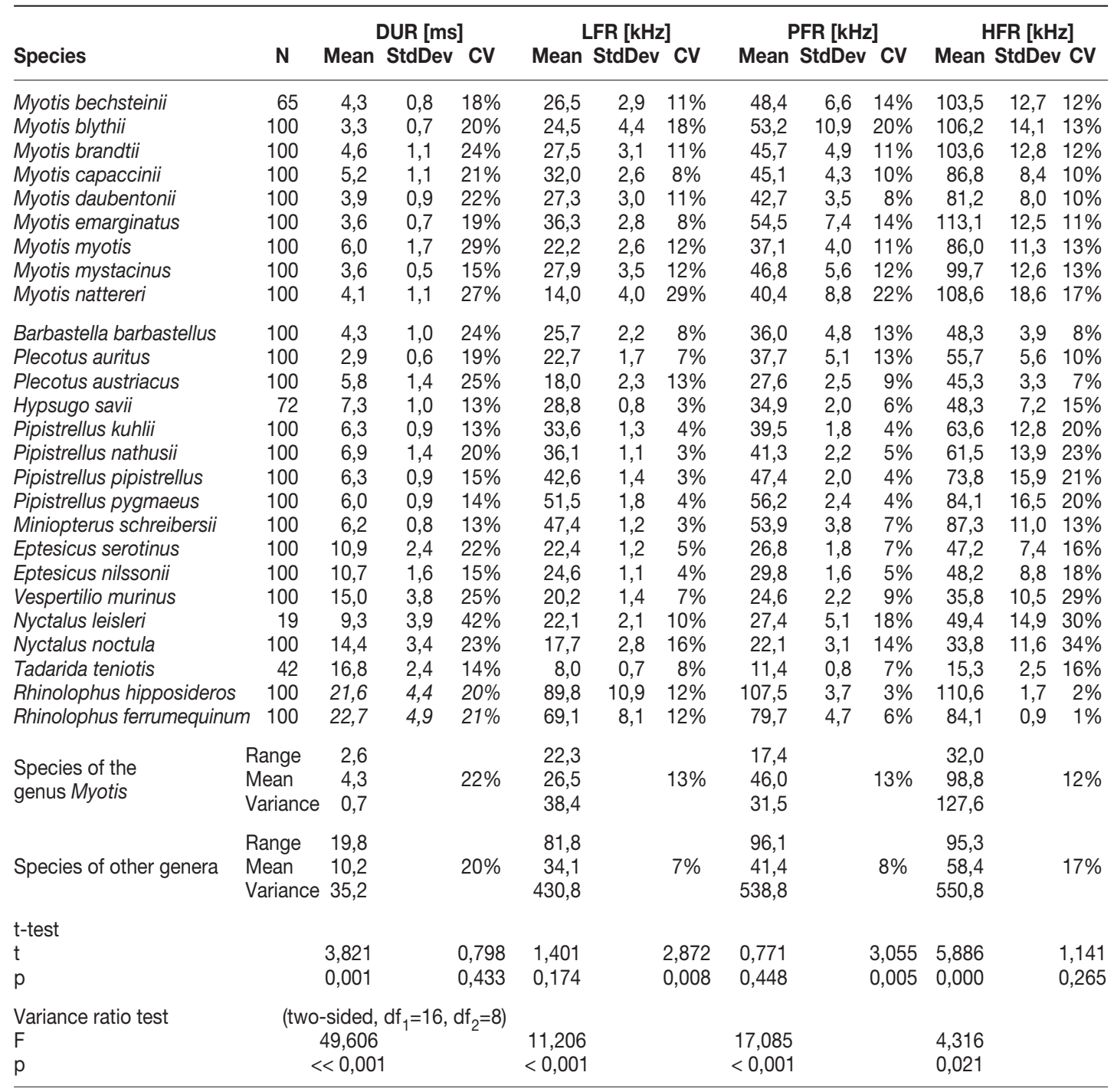

in our data set during recording. Thus, very short take-off calls were omitted from the analysis, thereby obviously influencing perceived variance. However, this was necessary to delimit the signals to only include the range of probable search calls. We thereby do not takle the task to identify species during e.g., approach or capture of insects but only within search flight. Recording bats of known identity differently, e.g., bats which hit the net after the recording, or recording them repeatedly, e.g., individuals which were lighttagged several minutes earlier, would improve the overall naturalness of the database, at the cost of a vastly increased sampling effort. 


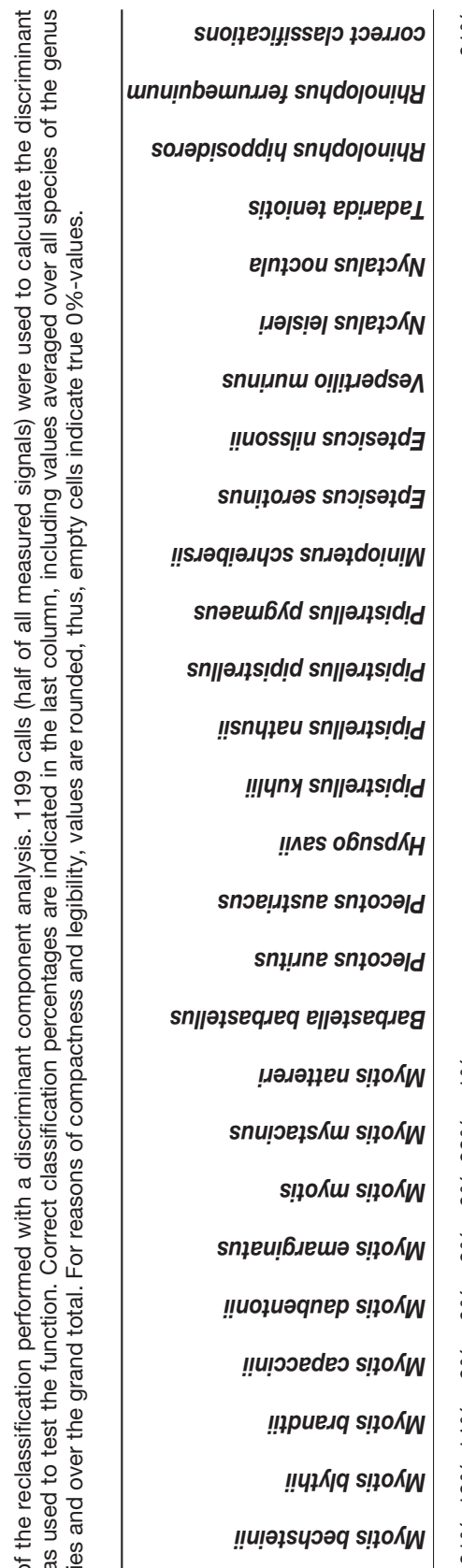

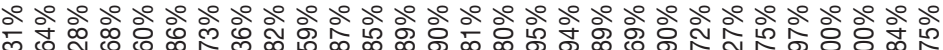
ㅇำ
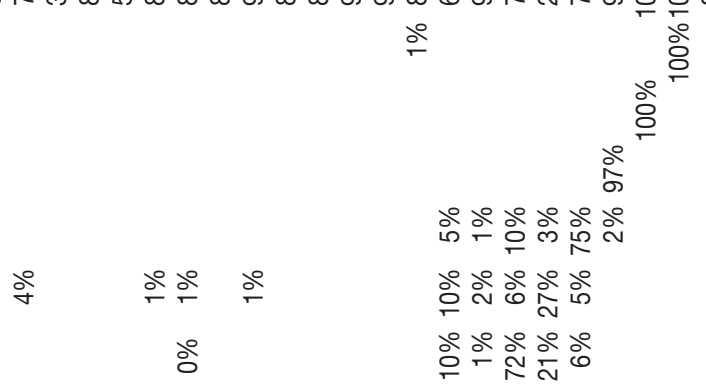

ลั

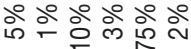

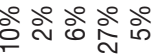

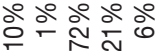

ㅇํㅇํำ ㅇํㅁ

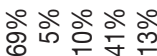

ㅇำ

离

ஃ̊

ㅇำ ㅇํㅇ

ํำ ㅇำ

ㅇํ ஃீ ஃீ

ㅇํㅇㅇㅇ ㅇํㅇ

ลे

ले ㅇํㅇ

ஃ̊ํำ

○े ㅇํㅇ

ㅇํํ ठ

ㅇํ ㅇํㅇ

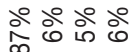

$\frac{1}{20}$

ㅇำ ㅇํㅇ

$\stackrel{2}{\circ}$

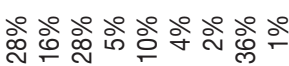

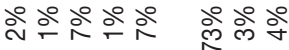

$\stackrel{1}{\circ}$

요 요

ஃ̊

ㅇํㅎ ㅇํㅇ

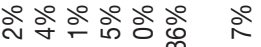

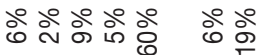

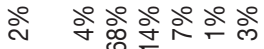

ㅇํ

ลे ลे ํํำ

ஓे

ปั

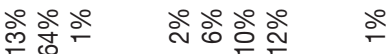

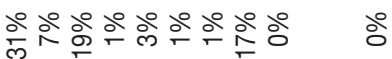

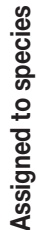

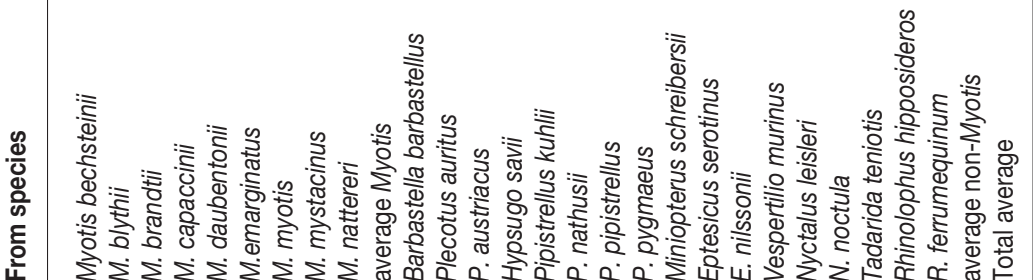


TABLE 3. - Results of the species classifications with the synergetic pattern recognition algorithm using 20 calls per each of the 26 species for learning. First four numeric columns give from left to right: the average recognition results for randomly selected training calls; recognition with a subselection from the random selection for training; recognition after rejecting signals with low match to any class and/or close match to several species, this again for the random sets and the subselection. Fifth to eighth numeric columns contain from left to right; the improvement effect of the subselection on raw and filtered classifications respectively, and the effect of filtering on the random set and subselection respectively.

\begin{tabular}{|c|c|c|c|c|c|c|c|c|}
\hline \multirow[b]{3}{*}{ Species } & \multicolumn{4}{|c|}{ classification } & \multicolumn{4}{|c|}{ effect of } \\
\hline & \multicolumn{2}{|c|}{ raw } & \multicolumn{2}{|c|}{ after filtering } & \multicolumn{2}{|c|}{ selection on } & \multicolumn{2}{|c|}{ filter on } \\
\hline & $\begin{array}{l}\text { random } \\
\text { selection }\end{array}$ & $\begin{array}{c}\text { sub- } \\
\text { selection }\end{array}$ & $\begin{array}{l}\text { random } \\
\text { selection }\end{array}$ & $\begin{array}{c}\text { sub- } \\
\text { selection }\end{array}$ & $\begin{array}{c}\text { raw } \\
\text { classifi- } \\
\text { cation }\end{array}$ & $\begin{array}{c}\text { filtered } \\
\text { classifi- } \\
\text { cation }\end{array}$ & $\begin{array}{l}\text { random } \\
\text { selection }\end{array}$ & $\begin{array}{c}\text { sub- } \\
\text { selection }\end{array}$ \\
\hline Myotis bechsteinii & $54 \%$ & $62 \%$ & $73 \%$ & $82 \%$ & $8 \%$ & $9 \%$ & $19 \%$ & $20 \%$ \\
\hline Myotis blythii & $63 \%$ & $67 \%$ & $82 \%$ & $79 \%$ & $4 \%$ & $-3 \%$ & $20 \%$ & $12 \%$ \\
\hline Myotis brandtii & $39 \%$ & $60 \%$ & $50 \%$ & $70 \%$ & $21 \%$ & $19 \%$ & $11 \%$ & $10 \%$ \\
\hline Myotis capaccinii & $54 \%$ & $60 \%$ & $75 \%$ & $67 \%$ & $6 \%$ & $-8 \%$ & $21 \%$ & $7 \%$ \\
\hline Myotis daubentonii & $40 \%$ & $43 \%$ & $60 \%$ & $75 \%$ & $3 \%$ & $15 \%$ & $20 \%$ & $32 \%$ \\
\hline Myotis emarginatus & $47 \%$ & $45 \%$ & $58 \%$ & $66 \%$ & $-2 \%$ & $8 \%$ & $11 \%$ & $21 \%$ \\
\hline Myotis myotis & $49 \%$ & $47 \%$ & $71 \%$ & $63 \%$ & $-2 \%$ & $-8 \%$ & $22 \%$ & $16 \%$ \\
\hline Myotis mystacinus & $52 \%$ & $58 \%$ & $73 \%$ & $78 \%$ & $6 \%$ & $6 \%$ & $20 \%$ & $20 \%$ \\
\hline Myotis nattereri & $60 \%$ & $55 \%$ & $87 \%$ & $86 \%$ & $-5 \%$ & $-2 \%$ & $28 \%$ & $31 \%$ \\
\hline Barbastella barbastellus & $66 \%$ & $70 \%$ & $86 \%$ & $84 \%$ & $4 \%$ & $-2 \%$ & $20 \%$ & $14 \%$ \\
\hline Plecotus auritus & $76 \%$ & $78 \%$ & $94 \%$ & $89 \%$ & $2 \%$ & $-5 \%$ & $18 \%$ & $11 \%$ \\
\hline Plecotus austriacus & $68 \%$ & $68 \%$ & $89 \%$ & $86 \%$ & $0 \%$ & $-3 \%$ & $21 \%$ & $18 \%$ \\
\hline Hypsugo savii & $89 \%$ & $95 \%$ & $95 \%$ & $97 \%$ & $6 \%$ & $1 \%$ & $6 \%$ & $2 \%$ \\
\hline Pipistrellus kuhlii & $78 \%$ & $85 \%$ & $93 \%$ & $91 \%$ & $7 \%$ & $-2 \%$ & $15 \%$ & $6 \%$ \\
\hline Pipistrellus nathusii & $70 \%$ & $68 \%$ & $81 \%$ & $84 \%$ & $-1 \%$ & $3 \%$ & $11 \%$ & $15 \%$ \\
\hline Pipistrellus pipistrellus & $88 \%$ & $90 \%$ & $97 \%$ & $98 \%$ & $2 \%$ & $2 \%$ & $9 \%$ & $8 \%$ \\
\hline Pipistrellus pygmaeus & $86 \%$ & $88 \%$ & $93 \%$ & $94 \%$ & $2 \%$ & $1 \%$ & $7 \%$ & $6 \%$ \\
\hline Miniopterus schreibersii & $79 \%$ & $85 \%$ & $90 \%$ & $92 \%$ & $6 \%$ & $2 \%$ & $11 \%$ & $7 \%$ \\
\hline Eptesicus serotinus & $61 \%$ & $68 \%$ & $75 \%$ & $87 \%$ & $7 \%$ & $13 \%$ & $13 \%$ & $19 \%$ \\
\hline Eptesicus nilssonii & $81 \%$ & $87 \%$ & $88 \%$ & $91 \%$ & $5 \%$ & $2 \%$ & $7 \%$ & $4 \%$ \\
\hline Vespertilio murinus & $64 \%$ & $75 \%$ & $79 \%$ & $96 \%$ & $11 \%$ & $16 \%$ & $15 \%$ & $21 \%$ \\
\hline Nyctalus leisleri & $97 \%$ & $98 \%$ & $98 \%$ & $100 \%$ & $1 \%$ & $2 \%$ & $1 \%$ & $2 \%$ \\
\hline Nyctalus noctula & $62 \%$ & $65 \%$ & $69 \%$ & $72 \%$ & $3 \%$ & $2 \%$ & $7 \%$ & $7 \%$ \\
\hline Tadarida teniotis & $97 \%$ & $100 \%$ & $100 \%$ & $100 \%$ & $3 \%$ & $0 \%$ & $3 \%$ & $0 \%$ \\
\hline Rhinolophus hipposideros & $94 \%$ & $93 \%$ & $97 \%$ & $98 \%$ & $-1 \%$ & $1 \%$ & $3 \%$ & $5 \%$ \\
\hline Rhinolophus ferrumequinum & $94 \%$ & $95 \%$ & $99 \%$ & $100 \%$ & $1 \%$ & $1 \%$ & $5 \%$ & $5 \%$ \\
\hline Total average & $70 \%$ & $73 \%$ & $83 \%$ & $86 \%$ & $4 \%$ & $3 \%$ & $13 \%$ & $12 \%$ \\
\hline Myotis average & $51 \%$ & $55 \%$ & $70 \%$ & $74 \%$ & $4 \%$ & $4 \%$ & $19 \%$ & $19 \%$ \\
\hline non-Myotis average & $80 \%$ & $83 \%$ & $90 \%$ & $92 \%$ & $3 \%$ & $2 \%$ & $10 \%$ & $9 \%$ \\
\hline
\end{tabular}

Temporal patterns of sound emissions, sometimes combined with spectral fluctuations, can be characteristic for some species and experienced observers are helped by this information. The information about the interval between signals was available to us, but of arguable quality. Single calls were automatically cut out from echolocation sequences based on a relatively simple intensity integration in the temporal signal. Weaker signals between louder ones could be missed, noise could be extracted as signal (later visually rejected) or calls of other bats would fall between the ones of the bat in focus (when recorded in front of a roost). Correspondingly, preliminary tests with a DFA including the interval did result in a decrease of recognition rate. We therefore did not include this information in our final analysis presented here. In a revised calculation the inclusion of the interval could be reconsidered in cases where the interval fulfills some plausibility testing, e.g., falling in a bandwidth which seems realistic for the species considered. Still, the value of this measure in automatically recognizing bats out of species assemblages hunting concurrently seems doubtful if it does not even become detrimental. 


\section{SIGNAL VARIANCES}

The variance in our data is a sample variance and we assume, that it is a good representation of the population variance, as we have recorded the same species' calls repeatedly on different occasions. However most of the recordings happened after handrelease, which leaves some insecurity regarding the population variance. This should be addressed in a follow-up project.

We quantified variation in the genus Myotis against that in other species. Variances of call parameters are not equal in all species. Species in the genus Myotis exhibit higher intra- and lower inter-species variance compared to the rest of the investigated species. This is most important for recognition tasks. Low-variance groups, which strongly differ from other groups, will be easily distinguished.

Several factors influence the observed variances. Our data set includes recordings of several individuals, which certainly differ in their call characteristics due to e.g., sex, age or size (Jones \& Kokurewicz 1994). Furthermore, a single individual has a variety of vocalizations it can emit, ranging from echolocation to social calls (Fenton 1985). The latter are not considered here, but echolocation signals are adjusted to the particular situation: the detection of a hard target on a longer range is optimally achieved with signals differing from those required for accurate location of a close object. Similarly, signals vary somewhat predictably, depending on a variety of factors as the location, specific surrounding and even conspecifics (e.g., Habersetzer 1981; Barclay et al. 1999). This variation does on one hand blur the picture; on the other hand, it offers new opportunities for the identification of behaviour or surrounding (see below).

Myotis sp. show a narrower spectrum of foraging approaches, mostly hunting at short ranges for air- or substrate-borne targets (Arlettaz 1996). The other groups additionally include species foraging on long ranges (e.g., Nyctalus noctula Zbinden 1989) or species homing in on fluttering targets (Rhinolophidae, Jones \& Rayner 1989). Signal theory demands different types of vocalizations for such tasks (Simmons \& Stein
1980), explaining one source for larger interspecies variance.

\section{SPECIES RECOGNITION}

Traditionally, identification of animal species is achieved by the taxation of morphometric characteristics like size, colour etc. Animal behaviour, especially communication, as a most often species specific characteristic with heavy selective value offers an alternative access to identification (Alcock 1979). Nocturnal terrestrial animals use either smell, bioluminiscence or sound to communicate. In diurnal taxation, acoustics are widely used in a variety of e.g., arthropod groups, amphibians and birds. Comparably, echolocating bats are conspicuous for conspecifics and other observers. However, their orientation signals have adapted to auto-communicate about the surrounding and therefore, in theory, only partially qualify for identification purposes. Nevertheless, bats occupy a variety of ecological niches and have evolved specific features in morphology, physiology and behaviour (Neuweiler 1984), which endorse the use of echolocation signals as species specific markers. We hypothesize, that acoustic species identification should be feasible.

\section{DATA REDUCTION}

Since the first publications on the use of echolocation calls for the identification of species (Ahlén 1981) the bioacoustic and electronic equipment available to bat workers has tremendously evolved. Different methods of data filtering, reduction and visualization (e.g., heterodyning detectors, count-down circuits and period-meters, respectively) can be applied in the field. Each has its eligibility in different situations (Parsons et al. 2000). The use of combined methods in a self-contained apparatus becomes more and more widespread and the risk of oversimplistically applying them is rising and controversially discussed (Barclay 1999; O'Farrell et al. 1999a, $1999 \mathrm{~b})$. The influence and experience of the observer will always be a dominant factor in such inventories, unless automated systems classify with decision trees (Herr et al. 1997) or artificial 
neural networks (Stocker 1998) on the basis of automated measurements.

\section{PARAMETRIC APPROACHES}

Parametric measurements taken e.g., from oscilloscopes, powerspectra or spectrograms allow for later analysis with a variety of approaches ranging from discriminant function analysis or DFA (Zingg 1990; Vaughan et al. 1997; Jones et al. 2000; Russo \& Jones 2002) to decision trees (Herr et al. 1997) and artificial neural networks or ANN (Stocker 1998; Parsons 2001). For measurements taken from data-reduced samples high recognition rates above $90 \%$ were reported for some species, others failed completely to be separated (Herr et al. 1997). Rather low recognition rates for the same data sample were achieved with ANN (Stocker 1998). Consistently, classifications (DFA or ANN) calculated on measurements read from high-quality recordings (not time expanded etc.) perform better: in a twelvespecies sample Parsons and Jones (2000) classified $79 \%$ correctly with DFA and $87 \%$ with ANN. Several other studies proved the ability of DFA to discriminate bat species: Zingg (1990) achieved $86 \%$ correct classification in 11 species, Vaughan et al. (1997) reached 89\% with 6 species, and Russo and Jones recently (2002) succeeded at separating eighteen vespertilionid species with $81.8 \%$ correct classification (range 38\%-98\%). Obrist et al. (2004) classified 60\% of all signals correctly. For a data sample comprising 26 species, we now correctly classified $75 \%$ of all calls tested. Thus, DFA to date seems a consistent and therefore promising approach to automated, off-line species identification.

But, unless automated and reliable extraction of call measurement can be performed, DFA is a time consuming task, which hampers its use in large monitoring programs.

\section{SyNERGETIC "REAL-TIME” CLASSIFICATION}

Only recently data processing of complete digital signals came in reach of real-time computing in the field. Complete signals invariably contain more information than any data reduced sample, translating into a higher potential for species identification. Learning from the experience of signals with comparable parameters but differing structure of the frequency sweep over time lead us to the conviction, that pattern recognition approaches should open a new facet to spectrogram classification. Synergetic pattern recognition has the big advantage of being able to process large data sets (in contrast to ANN) at a very rapid rate. The algorithm we use typically classifies several signals per second. Previous spectrogram calculation decreases this rate marginally. Even if the system lags behind real-time by the duration of the sample size and the time of analysis, depending on processor speed it reaches a duty-cycle of $10-20 \%$ in the field. The results presented here obviously were elaborated off-line. The classification results we achieved with this algorithm underline the results of the DFA: with preliminarily optimized sets of training patterns we achieved $86 \%$ correct classifications (74\% Myotis, 92\% non-Myotis), comparable but roughly 12 points better than the DFA $(75 \%$ overall reclassification success, 59\% Myotis, 84\% non-Myotis). Obviously parametric variances differing in Myotis and non-Myotis species equally affect both methods (Figure 5). The pattern recognition results differ in three Myotis species with the results previously published by Obrist et al. (2004). The later were obtained with limited data samples on these species and the resulting small variance has increased their recognition success. Nyctalus noctula scored worse in our data set for a similar reason: in the new data set more frequency modulated signals were included. In all cases, our new results are more trustworthy. For most species DFA underlines this with scores similar to the pattern recognition approach. The reason for the clear deviation of many Myotis species and Nyctalus leisleri remains unclear.

\section{AUTOMATED SPECIES IDENTIFICATION WITHIN REACH?}

So far, we have no control over the actual number of individual bats contained in the training base, only the number of randomly chosen signals. We will have to further investigate the composition of correctly and incorrectly classified 


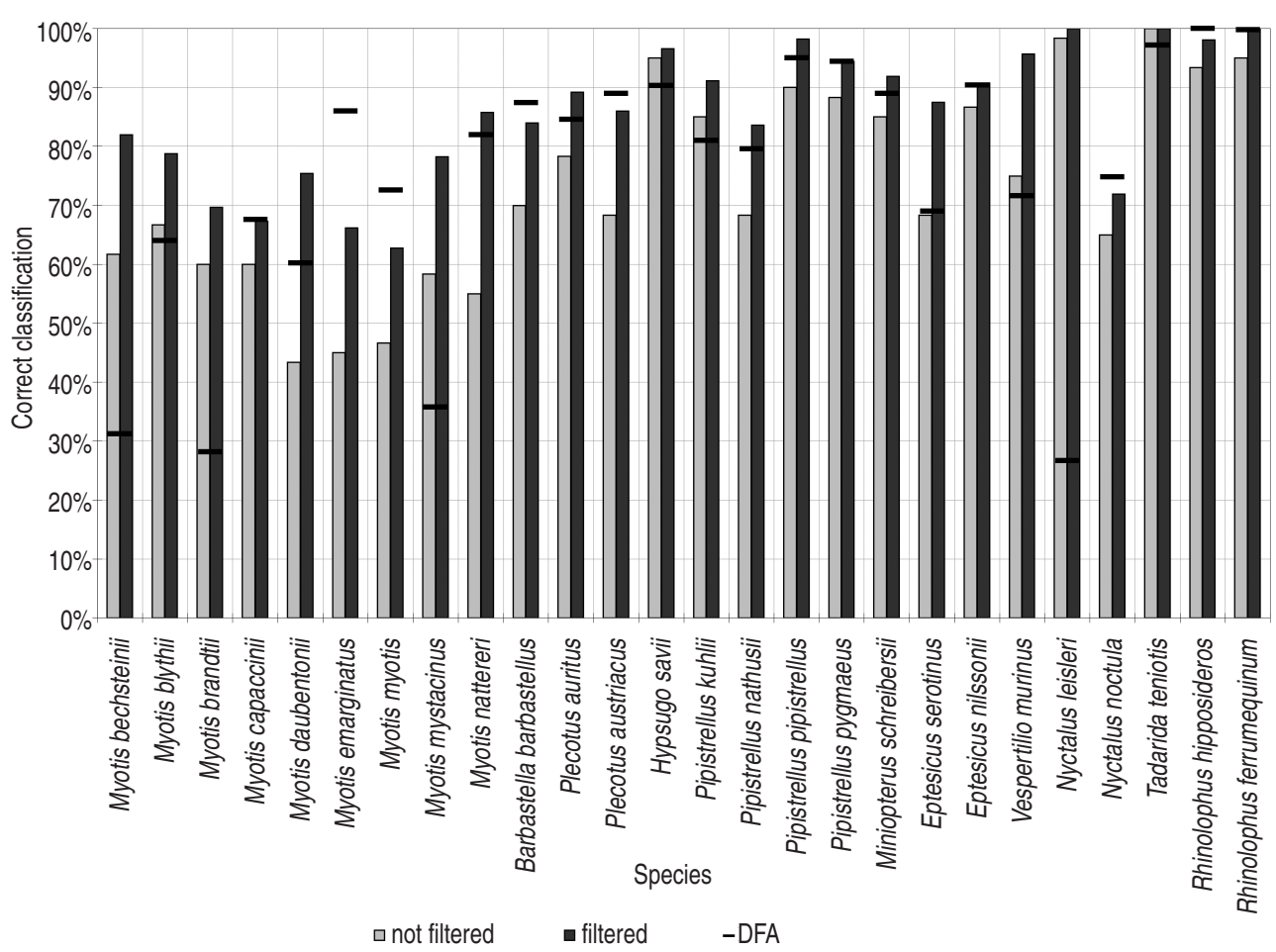

FIG. 5. - Classifications resulting from the synergetic pattern recognition algorithm. Correct classification percentages are given for: learning 20 calls of 26 species each, raw results (grey bars); dito but with filtering out of low probability classifications (see methods, black bars); black horizontal bars indicate the results from the discriminant function analysis (DFA).

signals. Do only single calls or full sequences (i.e. individual bats!) get misclassified?

Independent of this, quality, speed and autonomous operation seem to predestine our pattern recognition approach for automated monitoring of species specific bat activity. With computer speed increasing, the duty-cycle will increase, and we still see room for improvement in the recognition rate.

Random choice of training calls mixes broad- and narrow-band, long and short calls in a single species' prototype, hampering sharp classification. To decrease variance within groups in the future, we will differentiate each species' signals in three classes: those emitted close to clutter or during capture, those emitted in open space and a third intermediate group. Figure 3 shows comparable examples for species like Pipistrellus sp., Eptesicus sp. and others. Thus, by selecting many but similar calls with low variance per species, we hope to increase recognition success and at the same time get information about the behavioural situation of the bats present.

Repeated random selection of training signals from these species' sub-groups will allow us to evaluate those signals, that best represent the total and give further improved classification scores. Alternatively we evaluate methods to select calls systematically instead of randomly, iteratively replacing training calls, which degrade overall recognition success. Ultimately we may even be able to tackle individual recognition in species, which exhibit individual spectrogram characteristics as the ones given in Figure 6. Individual recognition is possible in some species (Obrist 1995) and may be of use for the estimation of population sizes (Burnett 2001). 


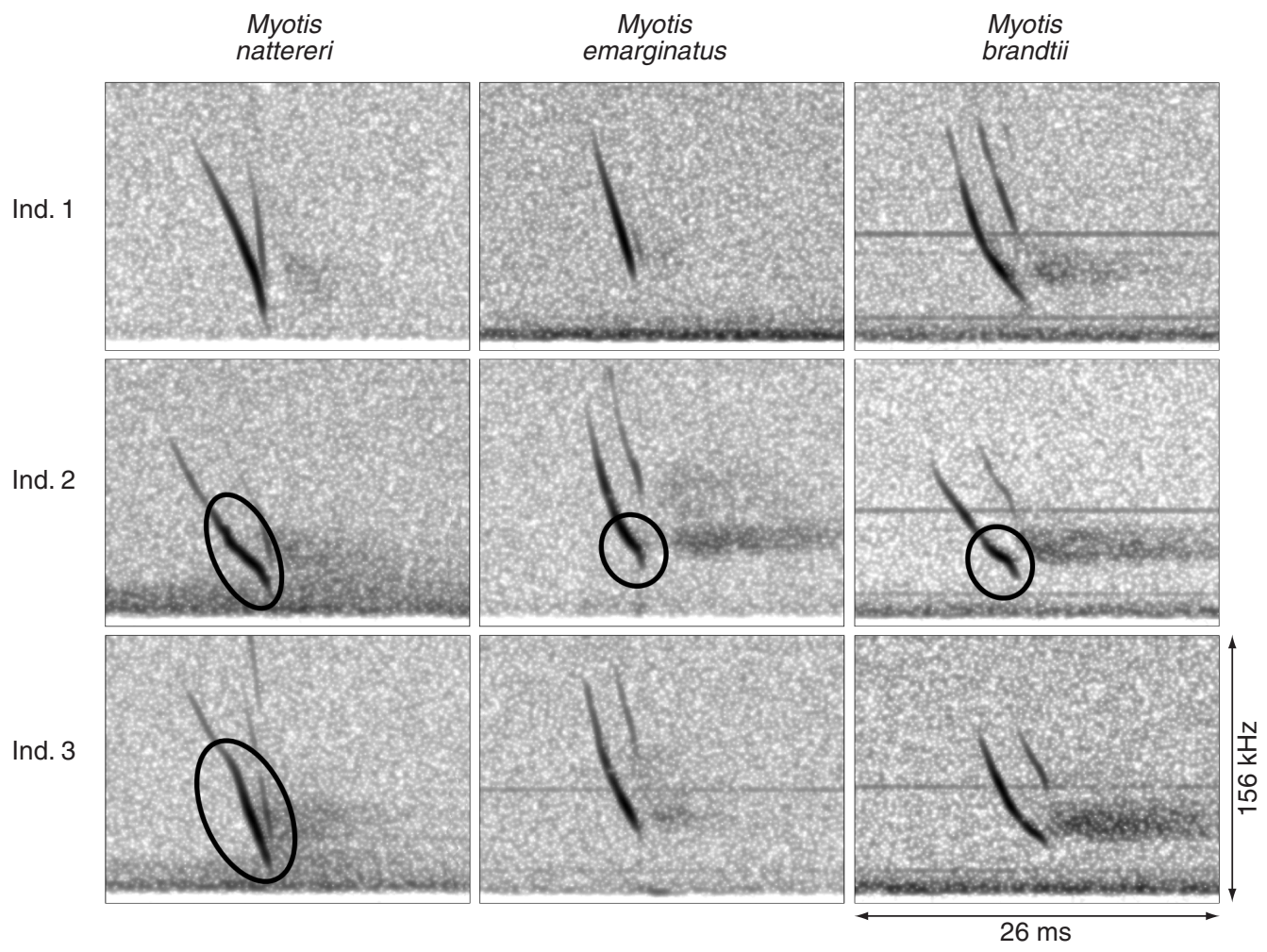

FIG. 6. - Spectrograms of three individual bats of each of three Myotis species, in which individual vocal signatures where found. The special "curvatures", which are emphasized in four sweeps with a black circle, appeared consistently over several calls in the corresponding individual.

\section{THE PROBLEM WITH PROBABILITIES}

Evidence of the presence of a species which has been unambiguously identified e.g., taxonomically or by its courtship song, is either existing or it is lacking. But albeit morphometric identification generally is assumed to be robust, examples of the contrary exist. Only recently have Pipistrellus pygmaeus (Barratt et al. 1997) and Plecotus alpinus (Kiefer \& Veith 2001) been separated from their sister species by evidence of genetic tests. The pipistrelles echolocation calls where known to differ in frequency by up to $10 \mathrm{kHz}$ (Zingg 1990), but morphometric differentiation is still tricky. The expected accuracy of the identification of a species of $100 \%$ is therefore not guaranteed in every case. Later revisions of the species may change detections to probabilities. This can seriously blur maps of species distribution or habitat use, as these maps rely on clear evidence of occurrence.

Furthermore, in some bat species echolocation calls are very diagnostic (e.g., Rhinolophidae). In others, e.g., Myotis sp., this is not the case. Different species exhibit different probabilities of detection (intensity of their calls) and of identification by acoustic means, independent of methods applied. Combined, this results in a species specific probability of evidence. If we want to apply monitoring methods with statistics or pattern recognition algorithms, we will have to deal with "probabilities of evidence of a species" occurrence, e.g., by adapting calculatory methods for generating e.g., distribution maps (Jaberg \& Guisan 2001). Still, if we are faced with the alternative of not being able to detect a species at all 
(e.g., by catching with nets), probabilistic approaches should be preferred.

Differential vocalization intensity, and consecutively chance of detection, can hardly be counterbalanced technically. But, by improving the training base through optimal call selection, we strive to increase at least probabilities of correct identification with our pattern recognition approach.

As a next step in the systems evaluation process we will crossvalidate the pattern recognition approach against standard methods like netting or acoustic surveys with heterodyning detectors (Kuenzi \& Morrison 1998; O’Farrell \& Gannon 1999), before we make the tool available to fellow bat researchers.

\section{Acknowledgements}

The project is supported by the Canton of Solothurn, the Swiss Agency for the Environment, Forests and Landscape (BUWAL) and the Swiss Bat Protection Foundation (SSF). We thank the many people who helped us catch and record bats.

\section{REFERENCES}

AHLÉN I. 1981. - Identification of Scandinavian bats by their sounds. The Swedish University of Agricultural Sciences, Department of Wildlife Ecology, Uppsala.

AlCOCK J. 1979. - Animal behaviour: an evolutionary approach. Sinauer Ass. Inc., Sunderland.

ARLETTAZ R. 1996. - Feeding behaviour and foraging strategy of free-living mouse-eared bats, Myotis myotis and Myotis blythii. Anim. Behav. 51: 1-11.

BARCLAY R. M. R. 1999. - Bats are not birds - A cautionary note on using echolocation calls to identify bats: a comment. J. Mammal. 80: 290-296.

Barclay R. M. R., Fullard J. H. \& Jacobs D. S. 1999. - Variation in the echolocation calls of the hoary bat (Lasiurus cinereus): influence of body size, habitat structure, and geographic location. Can. J. Zool. 77: 530-534.

Barratt E. M., Deaville R., Burland T. M., Bruford M. W., Jones G., Racey P. A. \& Wayne R. K. 1997. - DNA answers the call of pipistrelle bat species. Nature 387: 138-139.

BURNETT S. C. 2001. - Individual variation in the echolocation calls of big brown bats (Eptesicus fuscus) and their potential for acoustic identification and censusing. Ohio State University, Columbus.

DiECKMANN U. 1997. - SESAM: a biometric person identification system using sensor fusion. Pattern Recog. Lett. 18: 827-833.

Fenton M. B. 1985. - Communication in the Chiroptera. Indiana University Press, Bloomington.

FENTON M. B. 2000. - Choosing the "correct" bat detector. Acta Chiropt. 2: 215-224.

Fenton M. B. \& BelL G. P. 1981. - Recognition of species of insectivorous bats by their echolocation calls. J. Mammal. 62: 233-243.

Fenton M. B., Bouchard S., Vonhof M. J. \& Zigouris J. 2001. - Time-expansion and zerocrossing period meter systems present significantly different views of echolocation calls of bats. J. Mammal. 82: 721-727.

GRIFFIN D. R. 1958. - Listening in the dark. The acoustic orientation of bats and men. Yale University Press, New Haven.

Habersetzer J. 1981. - Adaptive echolocation sounds in the bat Rhinopoma hardwickei. J. comp. Physiol. 144: 559-566.

HAKEN H. 1988. — Learning in synergetic systems for pattern recognition and associative action. $Z$. Phys. B 71: 521-526.

HAKEN H. 1996. - Future trends in synergetics. Nonlinear physics of complex systems. Current status and future trends. Springer, Berlin.

Herr A., Klomp N. I. \& AtKinson J. S. 1997. Identification of bat echolocation calls using a decision tree classification system. Complexity International, 4. http://www.complexity.org.au /cilvol04/herr/batcall.html

HogG T. \& TAlHami I. 1996. - A competitive nonlinear approach to object recognition: the generalised synergetic algorithm, in Australian New Zealand Conference on Intelligent Information Systems, vol. 96, I.E.E.E., New York - Adelaide: 47-50

Jaberg C. \& GuISAN A. 2001. - Modelling the distribution of bats in relation to landscape structure in a temperate mountain environment. J. appl. Ecol. 38: 1169-1181.

Jones G. \& RAyner J. M. V. 1989. - Foraging behavior and echolocation in wild horseshoe bats Rhinolophus ferrumequinum and Rhinolophus hipposideros (Chiroptera, Rhinolophidae). Behav. Ecol. Sociobiol. 25: 183-191.

Jones G. \& KoKurewicz T. 1994. - Sex and age variation in echolocation calls and flight morphology of Daubenton's bats Myotis daubentonii. Mammalia 58: 41-50.

Jones G., Vaughan N. \& Parsons S. 2000. Acoustic identification of bats from directly sampled and time expanded recordings of vocalizations. Acta Chiropt. 2: 155-170.

KALKO E. K. V. 1995. - Insect pursuit, prey capture and echolocation in pipistrelle bats (Microchiroptera). Anim. Behav. 50: 861-880. 
Kiefer A. \& Veith M. 2001. - A new species of long-eared bat from Europe (Chiroptera: Vespertilionidae). Myotis 39: 5-16.

Kuenzi A. J. \& Morrison M. L. 1998. — Detection of bats by mist-nets and ultrasonic sensors. Wildl. Soc. Bull. 26: 307-311.

NeuWeILER G. 1984. - Foraging, echolocation and audition in bats. Naturwissenschaften 71: 446-455.

Neuweiler G. 1999. - Biology of Bats. Oxford University Press, New York.

OBRIST M. K. 1995. - Flexible bat echolocation: the influence of individual, habitat and conspecifics on sonar signal design. Behav. Ecol. Sociobiol. 36: 207-219.

Obrist M. K., Boesch R., Flückiger P. F. \& Dieckmann U. 2004. - Who's calling? Acoustic bat species identification revised with synergetics, in Thomas et al. (eds), Advances in the study of echolocation in bats and dolphins. Proceedings of the Biosonar Conference 1998. University of Chicago Press, Chicago.

O’Farrell M. J. \& Gannon W. L. 1999. - A comparison of acoustic versus capture techniques for the inventory of bats. J. Mammal. 80: 24-30.

O'Farrell M. J., Miller B. W. \& Gannon W. L. 1999a. - Qualitative identification of free-flying bats using the Anabat detector. J. Mammal. 80: 11-23.

O'Farrell M. J., Corben C., Gannon W. L. \& Miller B. W. 1999b. - Confronting the dogma: a reply. J. Mammal. 80: 297-302.

PARSONS S. 2001. - Identification of New Zealand bats (Chalinolobus tuberculatus and Mystacina tuberculata) in flight from analysis of echolocation calls by artificial neural networks. J. Zool., Lond. 253: 447-456.

Parsons S. \& Jones G. 2000. - Acoustic identification of twelve species of echolocating bat by discriminant function analysis and artificial neural networks. J. Exp. Biol. 203: 2641-2656.

Parsons S. \& Obrist M. K. 2004. - Recent methodological advances in the recording and analysis of chiropteran biosonar signals in the field, in Thomas et al. (eds), Advances in the study of echolocation in bats and dolphins, Proceedings of the Biosonar Conference 1998. University of Chicago Press, Chicago.

Parsons S., Boonman A. M. \& Obrist M. K. 2000. - Advantages and disadvantages of techniques for transforming and analyzing chiropteran echolocation calls. J. Mammal. 81: 927-938.

Russo D. \& Jones G. 2002. - Identification of twenty-two bat species (Mammalia: Chiroptera) from Italy by analysis of time-expanded recordings of echolocation calls. J. Zool., Lond. 258: 91-103.

Simmons J. A. \& STEIN R. A. 1980. — Acoustic imaging in bat sonar: echolocation signals and the evolution of echolocation. J. comp. Physiol. 135: 61-84.

STOCKER R. C. 1998. - The role of Artificial Neural Networks in the analysis of ultrasonic bat calls: a case study. Complexity International 5. http://www. complexity.org.au/ci/vol05/stocker/annbat $2 . \mathrm{htm}$

Titley Electronics 1998. - AnaBat II Bat Detector. Titley Electronics, Ballina. http://www. titley.com.au/tanabat.htm

Vaughan N., Jones G. \& Harris S. 1997. Identification of British bat species by multivariate analysis of echolocation call parameters. Bioacoustics 7: 189-207.

Wagner T., Schramm U. \& Boebel F. G. 1995. Synergetic learning for unsupervised texture classification tasks. Physica D 80: 140-150.

Wagner T., Boebel F. G., Hassler U., Haken H. \& SeITZER D. 1993. - Using a synergetic computer in an industrial classification problem, in Albrecht et al. (eds), International Conference on Artificial Neural Nets and Genetic Algorithms. SpringerVerlag, Berlin: 206-212.

Zar J. H. 1984. - Biostatistical Analysis. PrenticeHall Inc., Englewood Cliffs.

ZBINDEN K. 1989. - Field observation on the flexibility of the acoustic behaviour of the European bat, Nyctalus noctula (Schreber, 1774). Rev. suisse Zool. 96: 335-343.

ZINGG P. E. 1990. - Akustische Artidentifikation von Fledermäusen (Mammalia: Chiroptera) in der Schweiz. Rev. suisse Zool. 97: 263-294. 